\title{
Robert Eric Frykenberg, (éd.), Christians and Missionnaries in India. Cross-Cultural Communication since 1500
}

London, RoutledgeCurzon, 2003, 419 p.

Catherine Clémentin-Ojha

\section{OpenEdition}

Journals

Édition électronique

URL : http://journals.openedition.org/assr/19253

DOI : $10.4000 /$ assr. 19253

ISSN : $1777-5825$

Éditeur

Éditions de l'EHESS

Édition imprimée

Date de publication : 1 octobre 2008

Pagination : 163-274

ISBN : 978-2-7132-2192-7

ISSN : 0335-5985

\section{Référence électronique}

Catherine Clémentin-Ojha, « Robert Eric Frykenberg, (éd.), Christians and Missionnaries in India. CrossCultural Communication since 1500 », Archives de sciences sociales des religions [En ligne], 144 | octobredécembre 2008, document 144-29, mis en ligne le 04 février 2009, consulté le 21 septembre 2020. URL : http://journals.openedition.org/assr/19253 ; DOI : https://doi.org/10.4000/assr.19253 


\section{Robert Eric Frykenberg, (éd.), Christians and Missionnaries in India. Cross-Cultural Communication since 1500}

London, RoutledgeCurzon, 2003, 419 p.

Catherine Clémentin-Ojha

1 On a longtemps présenté les chrétientés et Églises de l'Inde comme autant d'extensions en terres lointaines des chrétientés et Églises d'Occident, mettant en relief les carrières des agents des organisations missionnaires. Toutefois, on a vu, ces dernières décennies, se multiplier des travaux d'historiens et d'anthropologues s'intéressant aussi aux conditions de réception du christianisme. Désireux de restituer le point de vue des convertis et non seulement celui des missionnaires, ces travaux montrent comment les groupes indiens christianisés se sont appropriés les pratiques et valeurs du christianisme en les accommodant à leur environnement culturel et social. Quoiqu'ils changent de point de vue en considérant surtout le sort des convertis, ils conservent pourtant de l'approche historique traditionnelle l'opposition irréductible entre «missionnaire» et "convertis ». L'ambition du volume collectif présenté ici est de sortir de cette opposition pour considérer la christianisation des Indiens comme le fruit d'une rencontre interculturelle. Les quinze contributions qu'il rassemble, sous la direction de l'un des meilleurs spécialistes de l'histoire des missions chrétiennes en Inde, montrent que le processus d'évangélisation ne peut se résumer à un transfert ou à une réception mais doit être envisagé comme une collaboration ou comme une construction commune. Elles mettent donc l'éclairage sur les Indiens christianisés qui, localement, servirent de relais aux missionnaires occidentaux (du simple point de vue numérique le personnel indigène fut bien plus important que ces derniers), ainsi que sur les différentes formes d'assistance que les missions reçurent des indigènes convertis et non convertis qui vivaient dans leur entourage. Elles examinent notamment l'apport de ces Indiens qui servirent d'informateurs, de catéchistes, de 
traducteurs. La majorité d'entre elles se concentre sur le grand moment missionnaire de l'Inde qui se produisit entre le $\mathrm{xIX}^{\mathrm{e}}$ et le $\mathrm{xx}^{\mathrm{e}}$ siècle, même si quelques-unes éclairent les périodes précédentes en situant historiquement leur propos. La plupart traite également de la partie méridionale du pays puisque, première christianisée, cette région a toujours rassemblé la plus forte concentration de chrétiens. Plutôt que de se livrer à un survol nécessairement rapide de chacune de ces contributions, on s'arrêtera sur la question qui sert de fil rouge au volume : celle de la relation entre évangélisation et colonisation.

2 Selon Robert Frykenberg, le maître d'œuvre du volume, « il n'y eut jamais au cours de l'histoire de l'Inde une majorité de missionnaires en Inde, britanniques ou non britanniques, qui se montrât prédisposée à être en faveur du colonialisme. » De fait les historiens restent assez divisés quand il s'agit d'évaluer la relation entre les principales nations qui colonisèrent l'Inde (le Portugal et la Grande-Bretagne) et les missions chrétiennes, certains estimant que ces dernières bénéficièrent dans l'ensemble du soutien des premiers, d'autres soulignant au contraire les obstacles que ceux-ci mirent sur leur route. En réalité, l'orientation radicalement différente des politiques religieuses portugaise et britannique en Inde empêche de caractériser la situation de manière générale. Est-on mieux à même de porter un jugement d'ensemble sur l'attitude des missionnaires à l'égard du fait colonial ? La complexité du lien entre évangélisation et colonisation apparaît lorsqu'on considère les circonstances dans lesquelles des Indiens aidèrent des Occidentaux à accumuler des savoirs sur la société indienne.

3 Les spécialistes des missions en Inde ont bien établi que les missionnaires mirent à profit leur présence dans le pays pour accumuler un grand nombre d'informations, contribuant ainsi, plus que tout autre groupe d'Occidentaux, à faire connaître au monde extérieur les langues, croyances et usages indiens. L'ouvrage confirme ce fait en montrant, à travers divers exemples bien choisis, qu'ils furent les premiers orientalistes et les premiers ethnologues, qu'ils rédigèrent les premiers dictionnaires et les premières grammaires. Ce faisant, il met clairement en lumière que ces savoirs, ils ne purent les acquérir sans l'aide assidue des Indiens : ils résultèrent de leur rencontre, de leur dialogue. Ainsi, par exemple, les missionnaires n'auraient pu apprendre et décrire les langues de l'Inde sans l'aide des lettrés indiens (convertis ou non). C'est une autre question de constater qu'ils passèrent les connaissances ainsi acquises au filtre de la science occidentale pour s'en servir. Ou d'observer que, de leur côté, les Indiens, loin d'être de simples récepteurs passifs, transformèrent aussi leurs propres traditions en les exposant aux Occidentaux dans un sens qui leur était favorable.

On admettra, avec les auteurs du volume, que les missionnaires n'avaient d'autre justification que d'annoncer l'Évangile et de faire entrer le plus grand nombre possible d'Indiens dans le giron de leurs Églises. Leurs savantes études se voulaient subordonnées à cette fin, même si leur démarche ne fut pas toujours comprise par leur milieu. Les autorités ecclésiales rechignèrent souvent à financer une telle accumulation de connaissances : ne fallait-il pas se consacrer au salut des âmes des païens plutôt que de perdre son temps à étudier leurs superstitions?

5 Mais comprendre les motivations des missionnaires, reconnaître même le talent, l'abnégation ou l'enthousiasme avec lesquels ils défrichèrent de nouveaux domaines d'études n'interdit pas d'observer la connivence entre leurs activités intellectuelles et l'entreprise coloniale. Car il est désormais bien établi que la colonisation de l'Inde ne 
résulta pas seulement d'une conquête militaire, économique, administrative et politique, elle fut également un phénomène intellectuel et culturel. En colonisant l'Inde, les Européens non seulement soumirent de larges sections de sa population à des formes inédites d'organisation politique et administrative, mais ils acquirent nombre de connaissances sur son histoire, sa géographie et ses cultures. Les administrateurs coloniaux eux-mêmes se livrèrent à de savantes études et se consacrèrent à la collecte d'informations de toutes sortes sur la société indienne. Certes les deux processus, domination politique et acquisition de savoirs, ne doivent pas être confondus même si on sait que, dans la réalité, ils se renforcèrent mutuellement. Et une telle distinction est d'autant plus requise s'agissant de ces Européens particuliers qu'étaient les agents des différentes congrégations missionnaires chrétiennes œuvrant en Inde. Mais il faut aussi reconnaître que le rapport était étroit entre information et conquête coloniale: l'ensemble des connaissances accumulées par les Occidentaux, par les missionnaires comme par les autres, servit à la puissance coloniale britannique pour établir et consolider son pouvoir sur l'Inde. Et même si les savoirs sur l'Inde résultent de la rencontre entre des lettrés indiens et occidentaux, et non de l'imposition des idées du second groupe sur le premier, ils ne se construisent pas moins dans l'espace colonial et dans la langue du groupe dominant, l'anglais. Il convient donc de qualifier le processus historique des interactions en question en gardant à l'esprit les circonstances historiques dans lesquelles elles se sont déroulées. C'est le contexte colonial qui les a rendues possibles: celui-ci fut la condition historique qui permit le dialogue des orientalistes, des administrateurs, des missionnaires avec des lettrés indiens.

Reste que penser l'évangélisation de l'Inde dans les termes d'une collaboration entre missionnaires et convertis autorise une présentation équilibrée de l'activité missionnaire en Inde qui va au-delà de sa caractérisation comme colonialiste. Elle revient aussi à poser, à nouveaux frais, une question qui occupe une large place dans l'historiographie contemporaine de l'Inde, celle du rôle des Indiens pendant la période coloniale. Au fond, il s'agit de redonner leur agency aux Indiens, du temps où ils étaient des sujets coloniaux. De souligner qu'ils n'ont pas davantage absorbé le christianisme de manière passive qu'ils n'ont été les spectateurs passifs des activités des colonisateurs de leur pays. Au contraire, ils ont interagi avec les missionnaires comme ils ont interagi avec les colonisateurs. Les articles du volume montrent qu'il y a dans cette approche ample matière à réflexion. 